\title{
Optical Disc and Blood Vessel Segmentation in Retinal Fundus Images
}

\author{
${ }^{1}$ Amira B. Sallow, ${ }^{2 H a w k a r ~ K h . ~ S h a i k h a ~}$ \\ ${ }^{1}$ Department of Computer Science, Nawroz University, Duhok, Kurdistan Region - Iraq \\ ${ }^{2}$ Department of Computer Science, Faculty of Science, Zakho University, Duhok, Kurdistan Region - Iraq
}

\begin{abstract}
Segmentation of optical disk (OD) and blood vessel is one of the significant steps in automatic diabetic retinopathy (DR) detecting. In this paper, a new technique is presented for OD segmentation that depends on the histogram template matching algorithm and OD size. In addition, Kirsch method is used for Blood Vessel (BV) segmentation which is one of the popular methods in the edge detection and image processing technique. The template matching algorithm is used for finding the center of the OD. In this step, the histogram of each RGB (Red, Green, and Blue) planes are founded and then the cross-correlation is founded between the template and the original image, OD location is the point with maximum cross-correlation between them. The OD size varies according to the camera field of sight and the resolution of the original image. The rectangle size of OD is not the same for various databases,

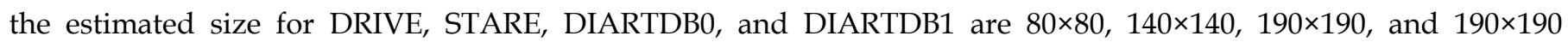
respectively. After finding the OD center and rectangle size of OD, a binary mask is created with Region of Interest (ROI) for segmenting the OD. The DIARTDB0 is used to evaluate the proposed technique, the result is robust and vital with an accuracy of $96 \%$.
\end{abstract}

Keywords: OD detection, OD ROI segmentation, template matching, Histogram matching, Blood Vessel segmentation.

\section{Introduction}

1 Optic disc (OD) detection and OD segmentation are the processes of localizing the OD center and detecting the boundary of OD respectively. Exact OD detection and OD segmentation are significant in ocular image analysis and computer-aided diagnosis for different types of eye diseases such as diabetic retinopathy (DR) and glaucoma [2]. OD recognized by color characteristics with intensity near to the light lesions. Thus, DR detecting needs to eliminate the OD pixels in

Academic Journal of Nawroz University

(AJNU) Volume 8, No 3 (2019).

Regular research paper: Published 11 June 2019

Corresponding author's e-mail : amo_bibo@yahoo.com

Copyright (C2018 1Amira B. Sallow, ${ }^{2}$ Hawkar Kh. Shaikha.

This is an open access article distributed under the Creative

Commons Attribution License. the retinal image. Fig. 1 shows the main components of the typical fundus image. The OD is the station of an appearance of the optic nerve and blood vessels that diversify into better vessels over the retina. Various forms of OD detecting techniques were proposed in the last decades. In general, the techniques are divided into two classes: in the first class, the process is built on characteristics of pixels that composed the OD such as form, color, and construction of OD. The second class of the techniques examines the retinal vasculature for finding the OD location. In the last decades, the proposed OD detection and segmentation methods have a number of typical common limitations. Primary, most reported OD detection methods fail to locate the exact OD center with an OD center. on the other hand, 
most of the segmentation methods do not remove the entire pixels within the OD and ROI. This instance affects the quality of detecting diabetic retinopathy and glaucoma. Histogram-based template matching is an efficient technique for detecting the center of the OD $[11,13]$.

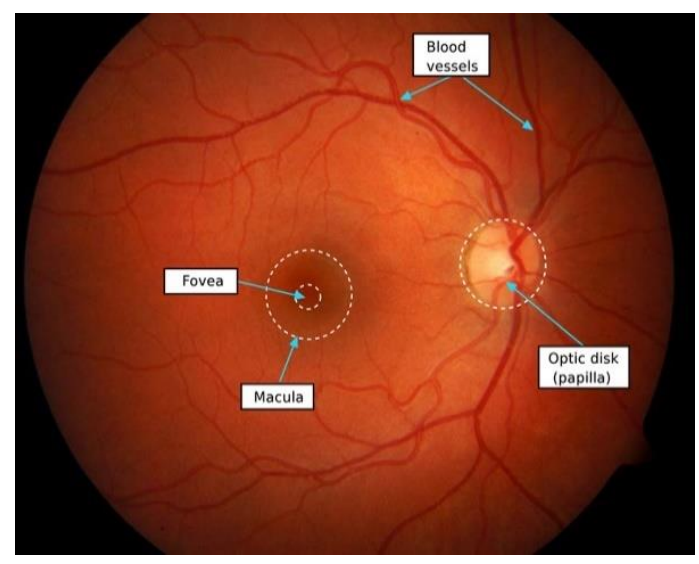

Fig.1. Main components of typical fundus image [7]

In this paper, the histogram template matching is applied for localizing the OD. In this technique, the histogram of a template is matched with the entire photos in the database to find the center of the OD. This process is the initial step for the segmentation process. Additionally, OD size is important to eliminate the exact ROI. The size of OD in a retinal image depends on Field of view (FOV) used by the camera and resolution of images. An approach for calculating the OD size in different databases using the FOV of the camera and image resolution is presented by in [15]. The estimated size for DRIVE, STARE, DIARTDB0, and DIARTDB1 databases are $80 \times 80,140 \times 140,190 \times 190$, and $190 \times 190$ respectively. After knowing the OD center and OD size, a binary mask is created to segment the OD in the retinal image. BV detection and segmentation are the second main process for DR detection since the intensity of the BVs are near to hemorrhages $(\mathrm{Hs})$ and microaneurysms (MAs) which are dark lesions. In this paper, Kirsch method is used for BV segmentation which is one of the popular methods in the edge detection and image processing technique [16].

The rest of this paper is organized as follows: In section II, we presented a literature review of the most recent papers. In section III, the methodology is presented. Next, we discussed the result in section IV, and then we concluded in the last section.

\section{Literature Review}

In this section, Various OD detection and segmentation strategies have been reported with the most resent techniques for BV segmentation in retinal fundus images. Most of the proposed diagnosis algorithms that use shape, color, and area for detecting DR. On the other hand, the detection of DR can be done indirectly by comparing fundus images of a patient's eye in different time intervals. The initial step of DR detection is $\mathrm{OD}$ and blood vessel detection and segmentation.

S. Lu [1] proposed a circular transformation to detect the circular form of the OD and the image variation crossways the OD borderline concurrently. In a certain region for every pixel of the image, the variation process is performed lengthwise several evenlyoriented external sections of definite length. A pixel is detected that has the largest variation lengthways entire external sections and is then used to detect the OD midpoint and the OD borderline.

S. Lu and J. H. Lim [9] used an inimitable circular brightness form related to the OD since the OD typically has a circular form and is usually brighter than the contiguous pixels, thus the concentration of pixels grow into darker according to their distance from the OD midpoint. They intended a line operator to detect brightness that has a circular form, which assesses the brightness variation of the image lengthways several line sectors of individual orientations that permit over all pixels. The variation with minimum/maximum used to locate the OD.

A. Aquino et al [10] proposed a template-based method 
for OD detection and segmentation. The technique utilizes morphological and edge detection methods with Hough Transform for circular shapes to obtain the OD borderline estimate. It indicates a single pixel belongs to OD as primary information. Thus, Votingtype algorithm is used for detecting pixel location.

In [12] A. Aquino et al, a combination of techniques is used for OD detection and segmentation. Firstly, they used three methods for localizing the Optical Disk Pixel (ODP) belongs to the OD. They used Maximum difference, Maximum variance, and Low-pass filter method separately for detecting ODP. Thus, three pixels are obtained, and the final ODP is the average of these locations. Next, for OD segmentation, they used a sequence of techniques. Firstly, a sub-image is obtained from the green channel of the RGB image, and then vessel removal and smoothing techniques are applied to the image, The Prewitt operator is operated to find a gradient magnitude image, a binary image is generated using Otsu thresholding, bleach version of the binary image is created using Morphological erosion, Lastly, the Circular Hough Transform is applied to find the circular approximation of the optic disc. "Fig. 2".

Template matching is another technique used by A. Dehghani et al [11] and P. R. Wankhede and K. B. Khanchandani[13]. in [11] A. Dehghani et al a histogram template is created from four first retinal images in DIVE database. The histogram is taken separately for each color, $\operatorname{Red}(\mathrm{R})$, Green(G), and Blue(B) of the retinal image. Next, they computed the average of the four histograms for composing the final histogram template for each color. Following, the template is used for scanning the OD location with the maximum correlation between the histogram of the template and the retinal image histogram. The process of OD segmentation, the best threshold is founded which is half of the maximum value of the correlation.
On the other hand, in [13] P. R. Wankhede and K. B. Khanchandani found that the histogram of OD ROI in the color images are almost the same. Therefore, only one template or more can be used for localizing OD with template matching.

In [14] A. Issac et al performed OD segmentation process using a new technique and cup depends on adaptive thresholding with characteristics of the color fundus image. The technique removes the information from the two channels of the image which are red and green channels by using mean and standard deviation for attaining a new image. The attained image includes "only the optic nerve head region in both the channels". The red channel is used for segmenting OD and the green channel used for segmenting optical cup. Next, the histogram of the preprocessed image is founded to determine a threshold.

In [16] P. Liskowski and K. Krawiec proposed a supervised segmentation technique that applies a deep neural network and it trained on a large dataset preprocessed with global contrast normalization, zero phase whitening, and enlarged using geometric transformations and gamma improvements. Numerous alternatives of the methods are considered, involving structured prediction, where a network classifies multiple pixels simultaneously. The technique learns from raw data pixels, in another word it does not depend on any previous information on vessel structure. Though learning, a network separately extracts thin features that are invariant to slight geometric deviations, then slowly transforms and combines them into higher-order features. "Fig. 3". In [17] an overlapping block-based system is proposed for retinal vessel detection by using support vector machine as classifier and standard deviation of the green channel intensity for the block, green intensity level for the pixel, average green intensity level of the 
block and sum of the 9 DCT coefficients from the antidiagonal and diagonal immediately below as feature vector. "Fig. 4".

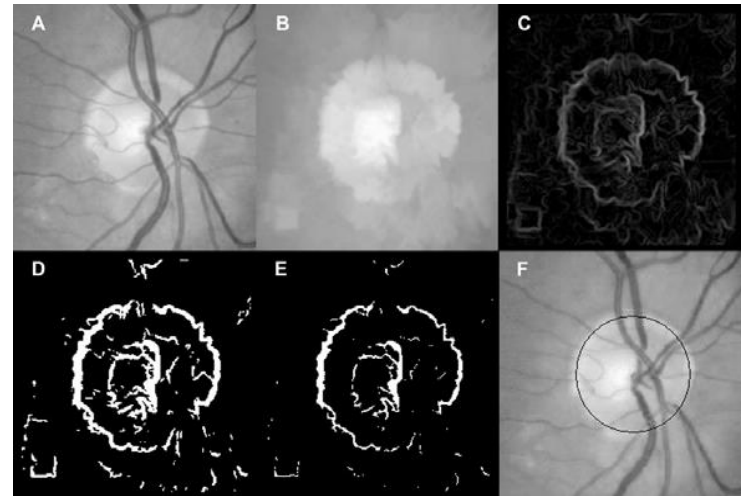

Fig. 2. OD segmentation process [12]:(A) sub-image obtained from the green channel of the RGB image, (B) vessel removal and smoothing technique, $(C)$ gradient magnitude image, (D) binary image, (E) bleach version of the binary image, (F) circular OD borderline approximate.

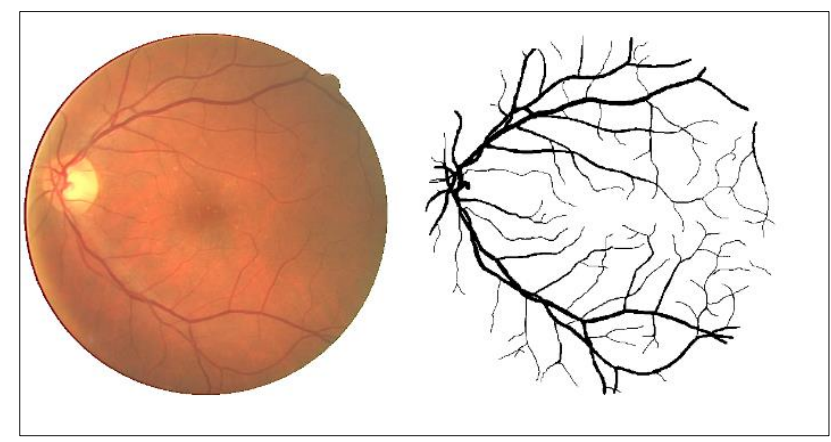

Fig. 3. An orginal image from the DRIVE database (left) and the equivalent manual segmentation of BVs (right)[16]

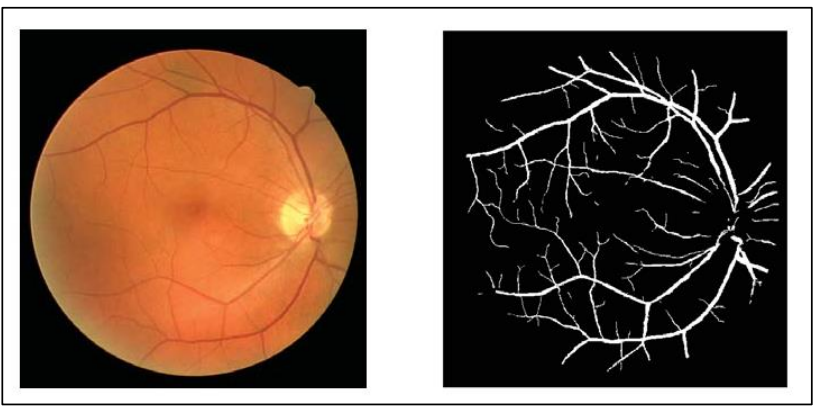

Fig. 4. An orginal image from the DRIVE database (left) and the equivalent manual segmentation of BVs (right)[17]

\section{Methodology}

In this section, the used database is discussed, and it followed by estimation technique to find OD size. Next, the template matching process was applied to localize the OD. Finally, the segmentation process is applied to the OD ROI.

\subsection{Databases}

In this study, DIARETDB0 database [6] is used for the assessment of projected OD detection and segmentation technique. The data is publicly accessible and relates to specific situations. A digital fundus camera is used for taking images with different levels of image noise optical out layers. Images were captured with a 50degree Field of View (FOV) with unknown camera settings. The data set includes 130 images of which 110 have signs of anomalies related to DR. In other words, 110 images contain signs of red small dots, $\mathrm{H}, \mathrm{HE}$, CWS or MA while 20 of them are regular with no sign of DR. The data related to practical circumstances and can be utilized to assess the general performance of analysis techniques. The data set is mentioned as "calibration level 0 fundus images". Furthermore, the database contains a folder including effective area masks. These masks are black and white images to remove noisy parts, out layers and background from retinal images. In this study, removing noisy region is part of the preprocessing by multiplying these masks with the original images. It should be renowned that excluding a few cases, the valid area includes the whole image and preprocessing does not effect on the details of the image. "Fig. 5" shows two example fundus images, their binary masks and resulted in noise-free images.

Another important information available by this data set is the Ground Truth (GT) file. GT has been arranged by medical specialists. For each image, a text file is accessible comprising five accesses each of them indicated one of the five kinds of anomalies which may exist in fundus images of patients sorrowing from DR. If a particular anomaly does not exist, its associated entry is registered as "n/a $\mathrm{a}$. If it does, the name of it i.e. $\mathrm{H}, \mathrm{HE}$, or NV etc. is written there.

\subsection{OD Size Assessment And ROI With the OD}

OD is assumed as a completely circular shaped 
segment with its center located on that pixel. The size of this circle in a retinal image determined by FOV utilized by the camera and resolution of images. In [15] a method is proposed to estimate the OD size depending on the FOV of the camera and image resolution. In TABLE 1 we illustrated the estimated size of OD corresponding to each database.
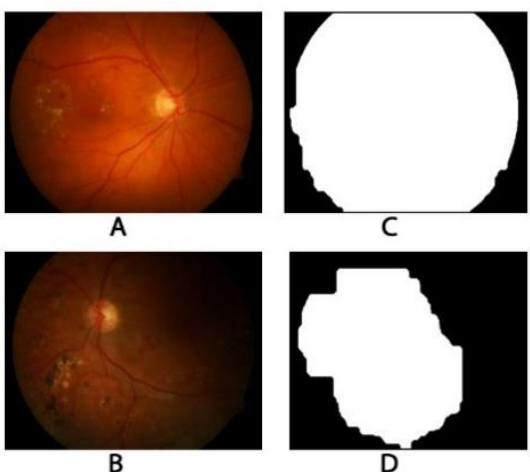

Fig. 5. Removing noisy region using binary masks $(A, B)$ Original fundus image $(C, D)$ binary noise removal mask $(E, F)$ noise-free image

\subsection{Histogram Template Creation}

A data template is created from first four images in the DIARETDB0 database. Histogram of each image is calculated separately, and then an average of the histogram is used as a data template. The histogram template creation starts with some preprocessing techniques such as remove the noise and eliminating out-layer data from the fundus images using binary masks. Thus, the input of this step is the original RGB retina image, and the output of this step is the noisefree fundus image. Firstly, the retina image is separated into R, G and B channels. In the second step, the mask is multiplied by each channel. After computing masked planes, the last step is concatenating them to produce a noise-free color image. "Fig. 5" shows the result of removing noise using binary masks. Afterward, the image is filtered with a 6X6 average filter to remove sharp edges and expands the contrast of the image. However, as fundus images are in the RGB format, each channel needs to be filtered separately. Next, the image is cropped in a 190X190 rectangular ROI with a centroid depending on the location of OD. The histogram is calculated for each channel separately (Red, Green, and Blue) and stored as a template. The cropped image and histogram of each channel are shown in "Fig. 6". A single template of first four images is used for detecting the OD for all images since the histograms of OD ROI are almost the same for all images within a specific database [13]. "Fig. 7" shows the histogram for Red channel for the different images.

TABLE 1. DIFFERENT DATABASES WITH IMAGE SIZE AND ESTIMATED SIZE FOR OD ROI [13].

\begin{tabular}{|l|c|c|}
\hline \multicolumn{1}{|c|}{ Database } & Image size & Rectangle size for OD ROI \\
\hline DRIVE & $768 \times 584$ & $80 \times 80$ \\
\hline STARE & $605 \times 700$ & $140 \times 140$ \\
\hline DIARTDB0 & $1500 \times 1152$ & $190 \times 190$ \\
\hline DIARTDB1 & $1500 \times 1152$ & $190 \times 190$ \\
\hline
\end{tabular}

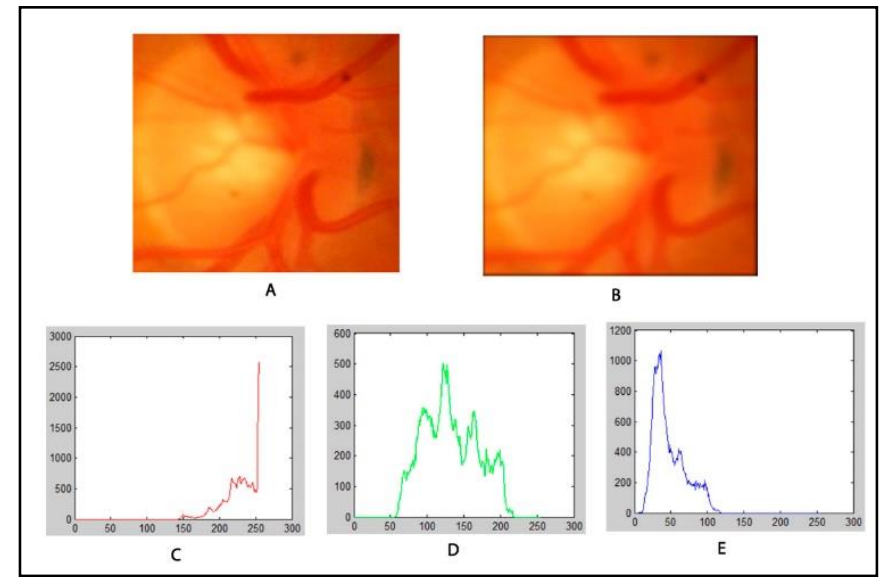

Fig. 6. Cropped OD ROI for Histogram template; (a) ROI holding OD candidate (b) Filtered OD candidate with average filter (c) (d) and (e) histogram of Red channel, Green channel and Blue channel respectively. 

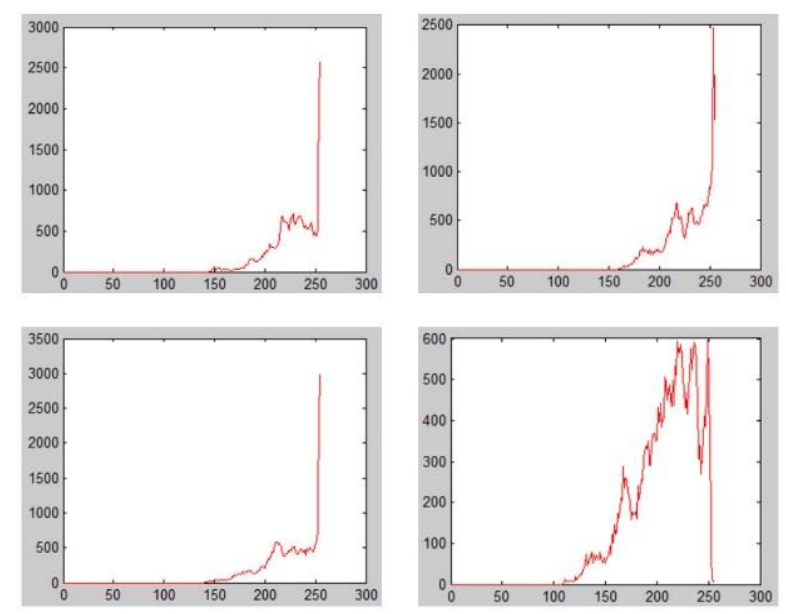

Fig. 7. Image histogram Red channel for four different fundus images

\subsection{OD Detection}

At the beginning of this stage, the image out layer and noise are removed using binary masks provided with the database. Next, the image is filtered with a $6 \times 6$ average filter to remove sharp edges and expands the contrast of the image. Then the searching process is performed to find the OD ROI, the image is segmented into rectangular blocks of fixed size 190X190 and via an iterative scheme, histogram of each block is founded and compared to the template histogram. The goal is to obtain a block with a maximum matching degree of the histogram with the template histogram. Calculation of similarity score is as follows: Initially, a block of size 190X190 is chosen from the filtered images in red, green, and blue channels. Next, the histogram is founded and a correlation coefficient is computed to each of them consistent with equation (1).

$$
\mathrm{C}_{\mathrm{p}}=\frac{1}{1+\sum\left(T_{p}-I_{p}\right)^{2}}
$$

where $p$ is either $R$ or $G$ or $B$ representing red, green or blue channel, $\mathrm{Tp}$ is the histogram of the template plane and Ip is the histogram of the selected block for the same color plane.

In this stage, three correlation coefficients, namely $\mathrm{Cr}$, $\mathrm{Cg}$, and $\mathrm{Cb}$ are obtained. The similarity score $\mathrm{C}(\mathrm{i}, \mathrm{j})$, is the weighted sum of these three coefficients. The pixel with a maximum value of $C(i, j)$ is the location of the optical disk. It should be noted that the contribution of the color planes in contrast to the OD image is not similar. Actually, the Green plane has the highest weight, the Blue plane has a moderate weight and the Red plane has the lowest weight. Subsequently, the weights are described as 2, 1 and 0.5 [13]. These weights are signed as $\mathrm{g}$, b, and r. Lastly, the total similarity score is calculated as in equation (2).

$$
C(i, j)=r \times C_{r}+g \times C_{g}+b \times C_{b}
$$

\subsection{OD ROI Segmentation}

The OD localization is the initial step for OD ROI segmentation. We presented a technique for segmenting ROI containing the OD. in this technique, the image is cropped in a circular form with a specific center and radius, and then a special binary mask is created as the following. The detected location of the OD $C(i, j)$ is used as the centroid of the circle, and the rectangular size of the OD ROI is used as the diameter of the circle. Firstly, we created two grids depending on the center and radius of OD using ndgrid MATLAB function [8]. Each grid is a two-dimensional array as shown in "Fig. 8". Next, the circular mask is created using equation (3).

$$
\operatorname{mask}(\mathrm{i}, \mathrm{j})=\left\{\begin{array}{cc}
1 & \left.\left.\mathrm{x}_{(\mathrm{i}, \mathrm{j})}\right)^{2}+\mathrm{y}_{(\mathrm{i}, \mathrm{j})}\right)^{2}<\mathrm{r}^{2} \\
0 & \left.\left.\mathrm{x}_{(\mathrm{i}, \mathrm{i})}\right)^{2}+\mathrm{y}_{(\mathrm{i}, \mathrm{i})}\right)^{2}>=\mathrm{r}^{2}
\end{array}\right.
$$

Where: $i=1,2,3, \ldots \ldots$, rows and $j=1,2,3, \ldots \ldots .$. , columns. the mask is the OD ROI mask, $x$ is the first grid, $y$ is the second grid that is created, $\mathrm{r}$ is the radius of the circle. In the next step, the binary mask is multiplied with the retinal image for segmenting the OD ROI.

\subsection{BV Segmentation}

BV detection and segmentation are the second main process for DR detection since the intensity of the BVs 
are near to Hs and MAs which are dark lesions. In this thesis, Kirsch method is used for BV segmentation which is one of the popular methods in the edge detection and image processing technique [150]. The method can be implemented quickly and it can detect thin BVs. The Kirsch method is consisting of 8 filters in $3 \times 3$ size. The filters are applied to the retinal image with 8 different angles from 0 and to 315 adding 45 degrees for each filter.

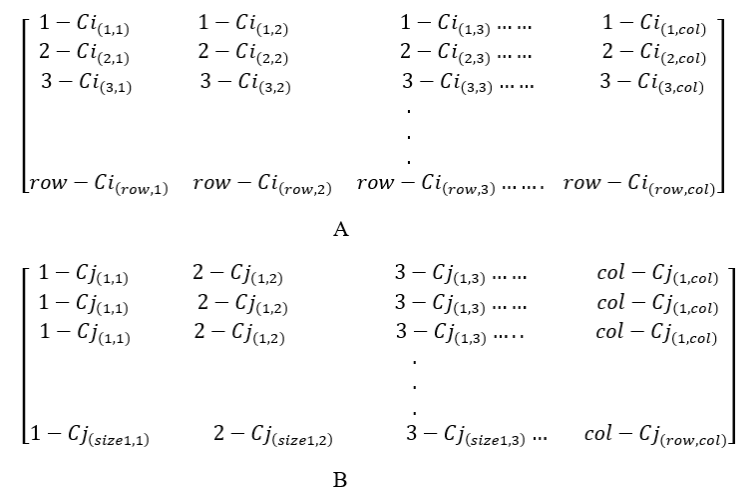

Fig. 8. A) The first grid depending on ' $x$ ' axis B) The second grid depending on ' $y$ ' axis

\begin{tabular}{|c|c|c|c|c|c|c|c|c|c|c|c|}
\hline$[-3$ & -3 & 5] & {$[-3$} & 5 & $5]$ & [ 5 & 5 & $5]$ & [5 & 5 & -31 \\
\hline-3 & 0 & 5 & -3 & 0 & 5 & -3 & 0 & -3 & 5 & 0 & -3 \\
\hline$[-3$ & -3 & 5] & {$[-3$} & -3 & $-3]$ & L-3 & -3 & $-3]$ & -3 & -3 & $-3]$ \\
\hline$[5$ & -3 & $-3]$ & {$[-3$} & -3 & $-3]$ & {$[-3$} & -3 & $-3]$ & {$[-3$} & -3 & 5] \\
\hline 5 & 0 & -3 & 5 & 0 & -3 & -3 & 0 & -3 & -3 & 0 & 5 \\
\hline L5 & -3 & $-3]$ & 5 & 5 & $-3]$ & 5 & 5 & $5]$ & [-3 & 5 & 5] \\
\hline
\end{tabular}

Fig. 9. Kirsch's template convolution kernels

The filter values are shown in Fig.9, and the degrees are $0,45,90,135,180,225,270$ and 315. The 8 filters are applied on the fundus images separately, and 8 images are gained with different edge enhancement result. This filter, depends on a predefined threshold value to detect the edges which is a number between 1 to 15 according to the dataset values. In this study, the Kirsch threshold value is indicated as 8 practically. The corresponding pixels of all enhanced images are compared and the maximum value is saved as the binary mask to segment the BVs. Fig. 10. Shows the result of BV segmentation using Kirsch's template and
Fig. 11. Shows the follow diagram of BV segmentation.

\section{Result}

In this paper, the entire pixels are removed after applying the technique for OD segmentation. In addition, the BVs are segmented which is the second preprocessing stage for DR detection. The result has experimented on 130 original images of DIARETDB0, the result was accurate and robust for \%96 of images. "Fig.12." shows the result of the proposed technique. The technique does not gain its goal to eliminate the exact ROI in two cases. First, when a misdetection of OD center occurs or the size of OD is abnormal caused by an illness. Second, when the OD ROI is not circular form, "Fig. 13".

We have compared the performance of our applied technique with methods presented earlier in terms of OD detection and segmentation success rate and execution time. It has been seen that our method is performing well and robust in the detection of OD. The time required for detection of OD is also compared to less than other methods regarding the size of images.

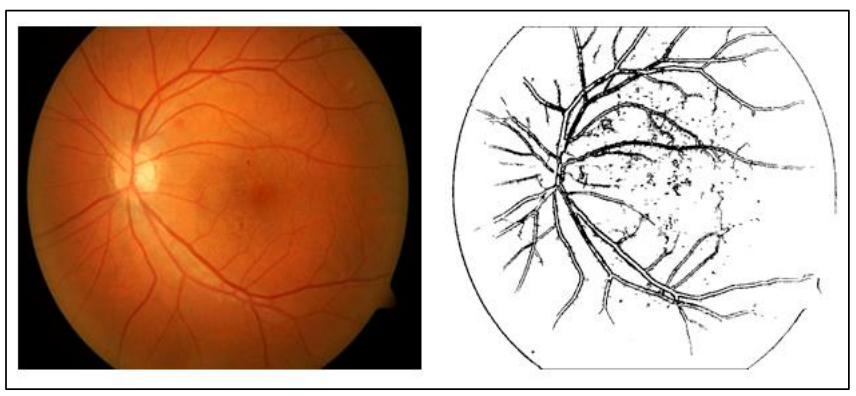

Fig. 10. BV segmentation using Kirsch's template. 


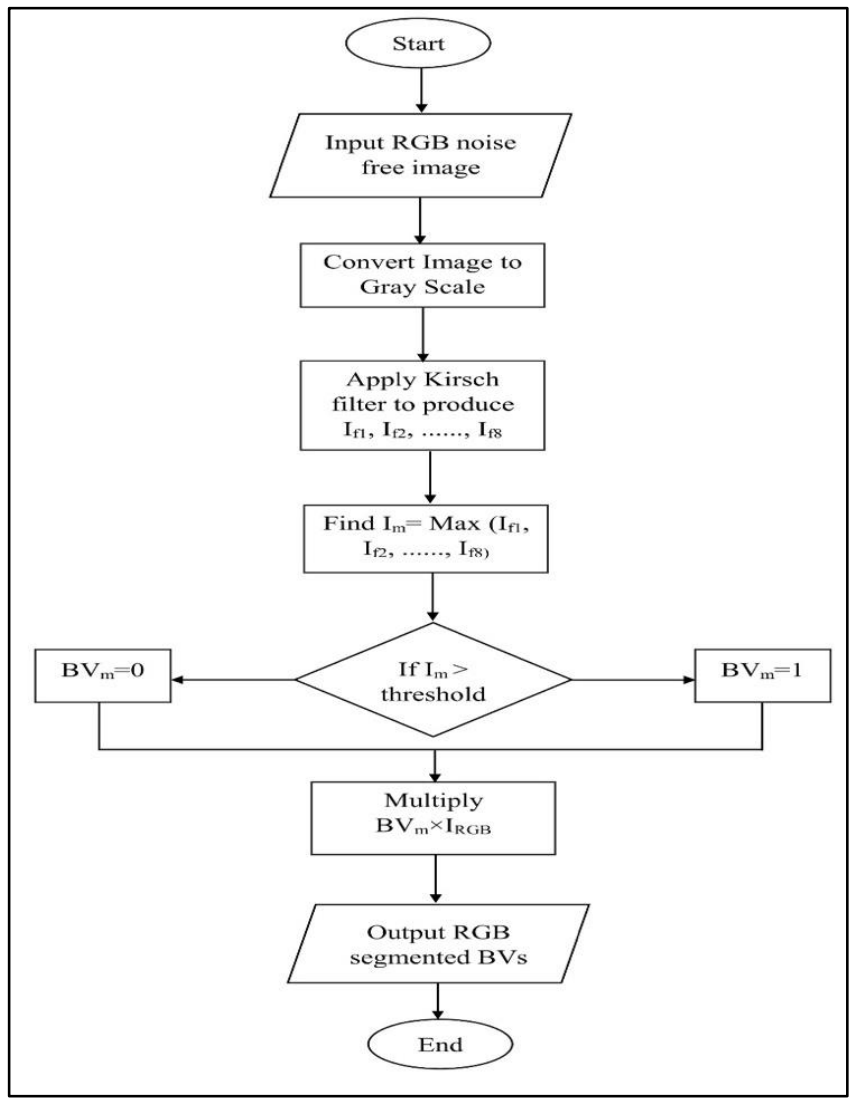

Fig. 11. Flow diagram of blood vessel segmentation.

TABLE 1. COMPARISON BETWEEN DIFFERENT TECHNIQUE FOR OD SEGMENTATION.

\begin{tabular}{|l|l|l|l|l|}
\hline Technique & $\begin{array}{l}\text { Success } \\
\text { rate } \\
(\boldsymbol{\%})\end{array}$ & Time(s) & Database & Image Size \\
\hline $\begin{array}{l}\text { Amin } \\
\text { Dehghani et } \\
\text { al [11] }\end{array}$ & $\begin{array}{l}100 \\
91.36 \\
98.9\end{array}$ & 27.6 & $\begin{array}{l}\text { DRIVE } \\
\text { STARE } \\
\text { Local }\end{array}$ & $\begin{array}{l}768 \times 584 \\
605 \times 700 \\
720 \times 576\end{array}$ \\
\hline $\begin{array}{l}\text { P. R. } \\
\text { Wankhede } \\
\text { et al [13] }\end{array}$ & 95 & 16.3 & DRIVE & $768 \times 584$ \\
\hline $\begin{array}{l}\text { H. Yu et al } \\
{[15]}\end{array}$ & 99 & 11.3 & $\begin{array}{l}\text { MESSID } \\
\text { OR }\end{array}$ & $1440 \times 960$ \\
\hline $\begin{array}{l}\text { Proposed } \\
\text { technique }\end{array}$ & 96 & 14 & $\begin{array}{l}\text { DIARET } \\
\text { DB0 }\end{array}$ & $1500 \times 1152$ \\
\hline
\end{tabular}
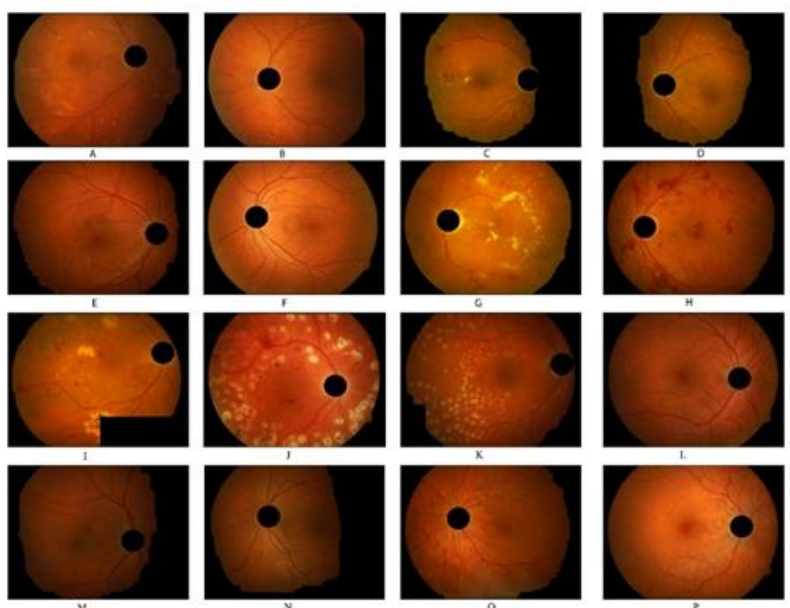

Fig.12. Segmentation result of the proposed technique
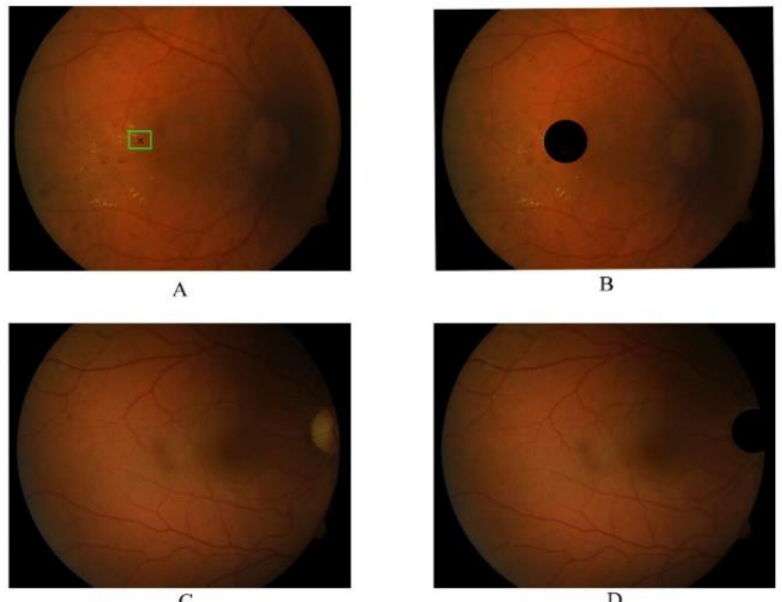

Fig.13. A) Misdetection of OD B) Segmentation of detected OD ROI

C) Elliptical OD

D) Circular segmentation of the OD

\section{Conclusion}

In this paper, a binary mask is applied for segmenting OD ROI. The technique is depending on the center of OD and the size of the OD ROI rectangle. Histogram template matching is used for detecting the center of the OD, and an estimated size of OD ROI rectangle $190 \times 190$ is used as the OD diameter. And then a binary mask is created depending on the OD center and the rectangle size of the ROI for segmenting the OD. In addition, Kirsch method is used to segment BVs in retinal fundus images. These thwo processes are initial step for DR detection in retinal images. The technique is applied to 130 images of DIARETDB0 database, the result is robust and vital with an accuracy of $96 \%$. 


\section{References}

1. S. Lu, "Accurate and Efficient Optic Disk Detection and Segmentation by a Circular Transformation," IEEE Trans. Med. Imaging, vol. 30, no. 12, pp. 21262133, 2011.

2. K. W. Tobin, E. Chaum, V. Priya Govindasamy, and T. P. Karnowski, "Detection of anatomic structures in human retinal imagery," IEEE Trans. Med. Imaging, vol. 26, no. 12, pp. 1729-1739, 2007.

3. E. J. Carmona, M. Rincón, J. García-Feijoó, and J. M. Martínez-de-la-Casa, "Identification of the optic nerve head with genetic algorithms," Artif. Intell. Med., vol. 43, no. 3, pp. 243-259, 2008.

4. A. A. H. A. R. Youssif, A. Z. Ghalwash, and A. A. S. A. R. Ghoneim, "Optic disc detection from normalized digital fundus images by means of a vessels' direction matched filter," IEEE Trans. Med. Imaging, vol. 27, no. 1, pp. 11-18, 2008.

5. A. A. A. Youssif, A. Z. Ghalwash, and A. S. Ghoneim, "Comparative Study of Contrast Enhancement and Illumination Equalization Methods for Retinal Vasculature Segmentation," Cairo Int. Biomed. Eng. Conf., no. DECEMBER, p. 5, 2006.

6. T. Kauppi, V. Kalesnykiene, J. Kamarainen, L. Lensu, and I. Sorri, "DIARETDB0: Evaluation Database and Methodology for Diabetic Retinopathy Algorithms," Mach. Vis. Pattern Recognit. Res. Group, Lappeenranta Univ. Technol. Finland., pp. 1-17, 2006.

7. M. Kuivalainen, "Retinal image analysis using machine vision," p. 96, 2005.

8. R. Sharma, S. Sharma, and M. T. Scholar, "Robust Watermarking of Color Images using RST Invariant Features," vol. 2, no. 6, pp. 142-149, 2014.

9. S. Lu and J. H. Lim, "Automatic optic disc detection from retinal images by a line operator," IEEE Trans. Biomed. Eng., vol. 58, no. 1, pp. 88-94, 2011.

10. A. Aquino, M. E. Gegúndez-Arias, and D. Marín, "Detecting the Optic Disc Boundary in Digital Fundus Images Using Morphological, Edge Detection, and Feature Extraction Techniques," IEEE Trans. Med. Imaging, vol. 29, no. 11, pp. 18601869, Nov. 2010.

11. A. Dehghani, H. Moghaddam, and M. Moin, "Optic disc localization in retinal images using histogram matching," EURASIP J. Image ..., pp. 1-11, 2012.

12. A. Aquino, M. E. Geg, and D. Mar, "Automated optic disk detection in retinal images of patients with diabetic retinopathy and risk of macular edema," Int. J. Biol. Life Sci., vol. 8, no. 2, pp. 87-92, 2012.

13. P. R. Wankhede and K. B. Khanchandani, "Optic disc detection using histogram-based template matching," in 2016 International Conference on Signal Processing, Communication, Power and Embedded System (SCOPES), 2016, pp. 182-185.

14. A. Issac, M. Parthasarthi, and M. K. Dutta, "An adaptive threshold based algorithm for optic disc and cup segmentation in fundus images," $2 n d$ Int. Conf. Signal Process. Integr. Networks, SPIN 2015, pp. 143-147, 2015.

15. H. Yu, C. Agurto, S. Echegaray, and M. S. Pattichis, "Fast Localization and Segmentation of Optic Disk in Retinal Images Using Directional Matched Filtering," no. May 2012.

16. P. Liskowski and K. Krawiec, "Segmenting Retinal Blood Vessels With _newline Deep Neural Networks," IEEE Transactions on Medical Imaging, vol. 35, no. 11, pp. 2369-2380, 2016.

17. E. Tuba, L. Mrkela, and M. Tuba, "Retinal blood vessel segmentation by support vector machine classification," 2017 27th International Conference Radioelektronika (RADIOELEKTRONIKA), 2017.

18. H. B. H. Bhadauria, "Vessels Extraction from Retinal Images," IOSR Journal of Electronics and Communication Engineering, vol. 6, no. 3, pp. 79-82, 2013 